\title{
Noninvasive system for evaluating allergen-induced nasal hypersensitivity in murine allergic rhinitis
}

\author{
Muneo Nakaya ${ }^{1}$, Makoto Dohi ${ }^{2}$, Katsuhide Okunishi ${ }^{2}$, Kazuyuki Nakagome ${ }^{2}$, \\ Ryoichi Tanaka ${ }^{2}$, Mitsuru Imamura ${ }^{2}$, Shintaro Baba ${ }^{1}$, Naonobu Takeuchi ${ }^{1}$, \\ Kazuhiko Yamamoto ${ }^{2}$ and Kimitaka Kaga ${ }^{1}$
}

${ }^{1}$ Department of Otolaryngology, Faculty of Medicine, University of Tokyo, Tokyo, Japan and ${ }^{2}$ Pulmonary Division, Department of Allergy and Rheumatology, Graduate School of Medicine, University of Tokyo, Tokyo, Japan

\begin{abstract}
Until now there has been no method for physiologically evaluating nasal hypersensitivity in mice. Enhanced pause (Penh) has been used as an indicator that reflects changes in the lower airway. Recently, however, there is disagreement regarding the significance of the Penh system; this is because Penh is not essentially a physiological parameter, and it might not necessarily represent a change in the lower respiratory tract. The purpose of the present study is to investigate whether Penh could be applicable for analyzing nasal hypersensitivity in mice. BALB/c mice were sensitized with ovalbumin (OVA) through a combination of intraperitoneal injection and daily intranasal challenge in an awake condition. Penh was measured at each time point during sensitization, or a serial change in Penh value was followed after the final nasal challenge and the effect of treatment was assessed. Following sensitization and nasal challenge, the Penh value gradually increased and showed a significant difference on day 14. Changes in IgE, eosinophil infiltration into nasal mucosa, and OVA-induced symptoms all strongly correlated with the increase in Penh. On day 19, after OVA nasal provocation, Penh gradually increased and reached maximal values 25 min after the challenge. Pretreatment with dexamethasone or a histamine $\mathrm{H} 1$ blocker significantly suppressed this increase in Penh. We confirmed that intranasal OVA challenge did not induce bronchoconstriction by measuring airway resistance and bronchoalveolar lavage fluid, and through histological examination. These results clearly demonstrate that Penh could be a useful noninvasive indicator for studying nasal hypersensitivity.
\end{abstract} Laboratory Investigation (2006) 86, 917-926. doi:10.1038/labinvest.3700452

Keywords: enhanced pause (Penh); nasal response; nasal hyper-responsiveness; nasal resistance; murine model; allergic rhinitis

Nasal obstruction is a common and important symptom of rhinitis. ${ }^{1}$ The inhalation of airborne allergens by patients with allergic rhinitis results in a congestion of the nasal passages that causes difficulty in nasal breathing and often complete nasal obstruction. ${ }^{2}$ In human beings, objective methods have been developed to assess nasal patency quantitatively. ${ }^{3}$ These methods include the measurement of nasal peak inspiratory flow and nasal peak expiratory flow, the peak flow nasal

Correspondence: Dr M Nakaya, MD, Department of Otolaryngology, Faculty of Medicine, University of Tokyo, Hongo 7-3-1, Bunkyo-ku, Tokyo 113-8655, Japan.

E-mail: muneo@interlink.or.jp

Received 21 February 2006; revised 11 June 2006; accepted 13 June 2006 patency index, ${ }^{4}$ anterior and posterior rhinomanometry, ${ }^{5}$ and acoustic rhinometry. ${ }^{6}$

The ability to reliably evaluate changes in nasal cavity patency in animals is of paramount importance in the study of pathophysiology and the preclinical evaluation of novel treatments for allergic rhinitis. So far, small animals such as rat $^{7}$ and guinea pig, ${ }^{8}$ or larger animals such as cat ${ }^{9}$ and dog, ${ }^{10}$ have been used to generate important information on nasal physiology and pathophysiology. Dynamic techniques require invasive procedures, and the experimental animal cannot survive. ${ }^{7,8}$ Static methods, such as acoustic rhinometry, that measure nasal cavity geometry independent of airflow have been used in only large animals in an unconscious condition. ${ }^{9,10}$ Recently mouse models have been employed, because in these animals various kinds of experimental interventions, such as adoptive cell 
transfer, backcrossing, transgenic procedures, and knockout techniques, are available. Therapeutic trials have also been perfomed in mice. However, until now there has been no way to evaluate changes in nasal cavity patency physiologically in mice because of the difficulty of the measurement of dynamic or static methods.

Enhanced pause (Penh) is a new indicator of lung function. It is a dimensionless parameter representing airway flow limitation. ${ }^{11-13}$ The main advantage of the Penh system is that animals can be handled in a conscious, unrestrained condition. Moreover, the reproducibility of the Penh value itself is high. Although Penh is a rather crude indicator of airway function compared with the precise physiological parameters, such as specific airway conductance (SGaw) or dynamic compliance (Cdyn), Hamelmann et $a l^{11}$ reported that Penh correlates well with the pulmonary resistance measured with conventional two-chamber plethysmography in anesthetized, unconscious animals under mechanical ventilation. This report supports the reliability of this parameter. So far, Penh has been used for measuring nonspecific bronchial hyper-responsiveness to bronchoconstrictive agents such as methacholine and acetylcholine. ${ }^{11-15}$

On the other hand, because Penh is a dimensionless marker that represents only a change in the pressure of the box chamber, factors besides inflammation in the lower airway tract could affect its value. Recent reports indicated that Penh does not necessarily have a good correlation with airway resistance; ${ }^{16,17}$ it could reflect airflow limitation in the upper airway, including the nasal cavity. However, no report has described an application of the Penh system to study nasal hypersensitivity.

The purpose of the present study was to investigate whether Penh could be applicable to the analysis of change in nasal hyper-responsiveness to a specific allergen. We prepared a murine model of allergic rhinitis by systemic immunization and nasal challenge with ovalbumin (OVA), then analyzed the nasal hyper-responsiveness through the use of the Penh system. We found that the change in the Penh value strongly correlated with the change in various parameters of allergic inflammation in the nasal airway. We also found that Penh precisely reflected the immediate-type hypersensitivity reaction provoked by the nasal allergen challenge. These results strongly suggested that this noninvasive system could be very useful in the study of nasal hypersensitivity.

\section{Materials and methods}

\section{Mice}

Male BALB/cAJcl mice (5 weeks of age) were obtained from CLEA Corp. (Tokyo, Japan) and housed in an air-conditioned room maintained at $22-24^{\circ} \mathrm{C}$ and $50^{\circ}-60 \%$ humidity. All the animal experiments carried out in this study were approved by the Animal Research Ethics Board of the University of Tokyo.

\section{Immunization of Mice and Nasal Challenge}

Male mice 5 weeks of age were immunized by an intraperitoneal injection of $2 \mu \mathrm{g}$ OVA (Worthington Biochemical Corp., Lakewood, NJ, USA)/2 mg of aluminum hydroxide gel on days 0 and 5 , followed by daily intranasal challenge (from day 12 to day 19) with $3 \%(\mathrm{w} / \mathrm{v})$ OVA diluted in $40 \mu \mathrm{l}$ of sterile normal saline $(20 \mu \mathrm{l}$ OVA solution, $2 \times$ per mouse). The control mice received saline injection and intranasal challenge instead of the OVA solution. The mice were subjected to the following evaluations on days $0,12,14$, and 19.

\section{Measuring of the Nasal Response by a Noninvasive Enhanced Pause System}

The nasal response to various drug or allergen challenges was measured in unrestrained, spontaneously breathing mice through barometric whole body plethysmography ${ }^{18}$ by using the Biosystem XA (Buxco, Troy, NY, USA) whole body plethysmography system, as reported previously. ${ }^{19-21}$ With this system, changes in the chamber pressure during the respiratory cycle of a mouse (during inspiration and expiration) are measured as an indicator of airflow obstruction. Briefly, the mice were placed in the main chamber (animal chamber) of the plethysmograph, and the pressure differences between this chamber and a reference chamber integral to the main chamber (termed the box pressure signal) were measured with a differential pressure transducer connected to the amplifier and were recorded. The box pressure signal is caused by volume and resultant pressure changes in the main chamber during the respiratory cycle of the mouse. From the box pressure signal, the phases of the respiratory cycle, tidal volumes, and enhanced pause (Penh) can be calculated. Penh is a dimensionless value that represents a function of the proportion of maximal expiratory to maximal inspiratory box pressure signals and of the timing of expiration. According to the manufacturer's instructions, Penh was calculated as $(\mathrm{Te}-\mathrm{Tr}) / \mathrm{Tr}$ (defined as 'pause') $\times(\mathrm{PEP} / \mathrm{PIP})$, where $\mathrm{Te}=$ expiratory time $(\mathrm{s})$; $\mathrm{Tr}=$ relaxation time (seconds), defined as the time of pressure decay to $30 \%$ of the total expiratory pressure signal (area under the box pressure signal at expiration); $\mathrm{PEP}=$ peak expiratory pressure $(\mathrm{ml} / \mathrm{s})$; and $\mathrm{PIP}=$ peak inspiratory pressure $(\mathrm{ml} / \mathrm{s})$. During the swelling of nasal mucosa, the main alteration in the pressure signal occurs during early expiration and leads to changes in the waveform of the box pressure signal. Penh reflects changes in the waveform of the box pressure signal during both inspiration and expiration and combines these changes 
with the timing comparison of early and late expiration (pause). Penh was measured for $2.5 \mathrm{~min}$ for each experiment. ${ }^{15}$

\section{Histamine-Induced Nasal Congestion and the Effect of Epinephrine Treatment in Naive Mouse}

In eight nonsensitized mice, nasal congestion was induced with $5 \%(\mathrm{w} / \mathrm{v})$ histamine delivered by intranasal drip ( $20 \mu \mathrm{l}$ solution, $2 \times$ per mouse). At $6 \mathrm{~min}$ after the histamine challenge, $0.0005 \%$ epinephrine was administered by intranasal drip (20 $\mu \mathrm{l}$ solution, $2 \times$ per mouse). Penh was followed during the experiment for the first $18 \mathrm{~min}$.

\section{Measurement of Total IgE and OVA-Specific IgE}

After the immunizations, blood samples were obtained from some mice and the sera were assayed for total IgE titers. The mice were anesthetized by an intraperitoneal injection of sodium pentobarbital (50-60 $\mathrm{mg} / \mathrm{kg}$ body weight). The abdominal wall was dissected and a blood sample was obtained with a 26-gauge needle from the inferior cava vein. After the samples had fully coagulated, they were centrifuged $\left(2000 \mathrm{~g}\right.$ for $20 \mathrm{~min}$ at $4^{\circ} \mathrm{C}$ ), and the sera were collected and stored at $-70^{\circ} \mathrm{C}$ until use. Total IgE (BD Pharmingen, San Diego, CA, USA) was measured with an ELISA kit. Briefly, antimouse IgE monoclonal antibody was coated on a 96-well plate by incubation at $4{ }^{\circ} \mathrm{C}$ overnight. After it was blocked with PBS with $10 \%$ FBS, $60 \mu \mathrm{l}$ of serum sample or standard solution was added to each well. The standard solution was diluted to $10,1,0.1,0.01$, and $0.001 \mu \mathrm{g} / \mathrm{ml}$, and the samples and standard dilutions were incubated in the plate at room temperature for $1 \mathrm{~h}$. After the plate had been washed thrice with PBS with $0.05 \%$ Tween 20 and antimouse IgE antibody solution had been added, the plate was incubated at room temperature for $2 \mathrm{~h}$. After a washing, avidinbiotin reagent solution was added and incubation was continued at room temperature for $1 \mathrm{~h}$. After this, substrate solution was added to the wells and the absorbance at $405 \mathrm{~nm}$ was measured after the plate had been allowed to stand at room temperature for 5 to $20 \mathrm{~min}$. The concentrations in the samples were calculated from a standard curve. To measure OVA-specific IgE in sera, the plates were coated with $1 \mathrm{mg} / \mathrm{ml} \mathrm{OVA} / 0.1 \mathrm{M}$ carbonate solution instead of captured Abs for IgE; then the ELISA kit was applied.

\section{Infiltration of Eosinophils in Nasal Mucosa}

The experimental mice were killed after being anesthetized by an intraperitoneal injection of sodium pentobarbital (50 to $60 \mathrm{mg} / \mathrm{kg}$ body weight). The decapitated heads were fixed in $10 \%$ formaldehyde $48 \mathrm{~h}$ at $20^{\circ} \mathrm{C}$. Specimens were then decalcified
7 days in 20\% EDTA-Na and embedded in paraffin. The paraffin-embedded nasal cavity was transversely sectioned at the level of the maxillary sinus by continuous microtoming at a thickness of $4 \mu \mathrm{m}$, and specimens were then deparaffinized and stained with hematoxylin-eosin. Eosinophils were counted by light microscopy. The number of eosinophils was quantified per unit $\left(1 \mathrm{~mm}^{2}\right)$ of epithelial basement membrane to a uniform depth of submucosa by using an eyepiece reticule. ${ }^{22}$ The average of cells counted in at least six sections was used for data analysis. The data were obtained from eight mice per group on day 0 , day 19 , and $24 \mathrm{~h}$ after final challenge.

\section{Evaluation of Allergen-Induced Nasal Symptoms}

Nasal symptoms were evaluated by counting the number of sneezes and nasal itching motions (nasal rubbing) that occurred within a 10-min interval after daily OVA intranasal provocation. Observations were performed in a cage $(60 \times 40 \times 30 \mathrm{~cm})$.

\section{Changes in Penh Value in the Immediate-Type Hypersensitivity Reaction in the Nasal Airway}

On day 19, after the eighth nasal OVA challenge, Penh was serially measured for $35 \mathrm{~min}$. Furthermore, to test the effect of drug treatment on Penh value, some challenged mice received intraperitoneal injections of dexamethasone $(20 \mathrm{mg} / \mathrm{kg})$ or d-chlorpheniramine maleate $(40 \mathrm{mg} / \mathrm{kg}) 30 \mathrm{~min}$ before the OVA challenge. Then, Penh was followed.

\section{Bronchoalveolar Lavage Analysis}

After the mice were anesthetized with pentobarbital, the lungs were lavaged four times with physiologic saline $(0.5 \mathrm{ml}$ each). Approximately, $1.7 \mathrm{ml}$ of the instilled saline was consistently recovered with gentle handling. Total cell numbers were counted with a hemocytometer. The cells were resuspended again into $1 \mathrm{ml}$ of saline with $1 \%$ BSA. Cytospin samples were prepared by centrifuging the suspensions (100 or $150 \mu \mathrm{l})$ at $300 \mathrm{rpm}$ for 5 to $10 \mathrm{~min}$. To clearly distinguish eosinophils from the neutrophils, three different stains were applied: Diff-Quick stain, May-Giemsa stain, and Eosino (Hansel) stain. On the basis of the findings with these stainings, cell differentials were counted with at least 300 leukocytes in each sample. The cell types were judged according to standard hemocytologic procedures as neutrophils, eosinophils, lymphocytes, or other mononuclear leukocytes (macrophages and monocytes). Five mice were used on day 0 , day 18, day 19, and day 20 (24 h after final challenge). 
Measuring Airway Resistance of the Lower Airway Tract and Examination of Lung Tissue

Lower airway resistance was assessed by a measurement of airway resistance (Raw) as reported previously. ${ }^{19}$ Briefly, anesthetized mice were tracheostomized and connected to a MiniVent ventilator (Hugo Sachs Elektronik, March, Germany), then ventilated with a tidal volume of $250 \mu \mathrm{l}$ and a respiratory frequency of 120 breaths/ min. The mice were placed inside whole-body plethysmographs (Buxco) to measure Raw, which was measured, before the nasal allergen challenge on day $19(n=5), 20 \mathrm{~min}$ after it $(n=5)$, and $24 \mathrm{~h}$ after it $(n=5)$ on day 19.

To investigate the inflammation of the lower airway in murine allergic rhinitis, the histopathology of lung tissue was examined. The lung was taken from the experimental mice before, $20 \mathrm{~min}$ after the final OVA challenge, or $24 \mathrm{~h}$ after the final OVA challenge on day 19, fixed with neutralized buffered formalin, and embedded in paraffin. Thick sections $(4 \mu \mathrm{m})$ were stained with hematoxylineosin. The intensity of bronchial inflammation was assessed.

\section{Data Analysis}

The data were expressed as mean \pm s.e.m. A statistical analysis was evaluated using two-way analysis of cariance (ANOVA) followed by the Student's $t$-test for comparison between two groups; a critical value $<0.05$ was considered significant.

\section{Results}

\section{Change in Penh after Histamine and Epinephrine Treatment in Normal Mice}

First we examined whether histamine delivered into the nasal passage could affect the Penh value. In a preliminary study, we investigated the effect of a solution remaining in the nasal cavity just after intranasal challenge by examination of the Penh after an intranasal delivery of saline in naive mice. Penh increased slightly for only $1 \mathrm{~min}$ after nasal challenge (the mean Penh value was $0.53 \pm 0.25$ ) and soon decreased to the earlier value (the mean Penh value was $0.36 \pm 0.21$ ). This result indicated that a nonspecific effect of drip liquid persisted only for a very short time. Histamine provoked a rapid increase in Penh within the first $2 \mathrm{~min}$. Penh in these mice remained above the baseline level for the next $18 \mathrm{~min}$ (Figure 1). The histamine-induced increase in Penh was almost completely reversed $3 \mathrm{~min}$ after the administration of epinephrine to the nasal passages. We confirmed that the intranasal administration of histamine did not provoke bronchoconstriction of the lower respiratory tract by histology (data not shown). This result indicated

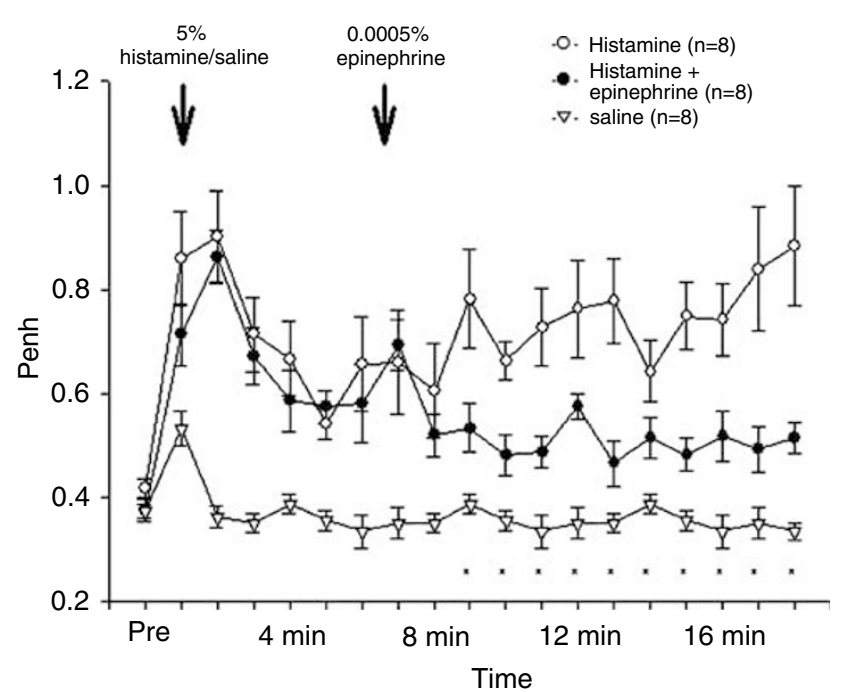

Figure 1 Histamine-induced nasal hyperresponsivness in naïve mouse.

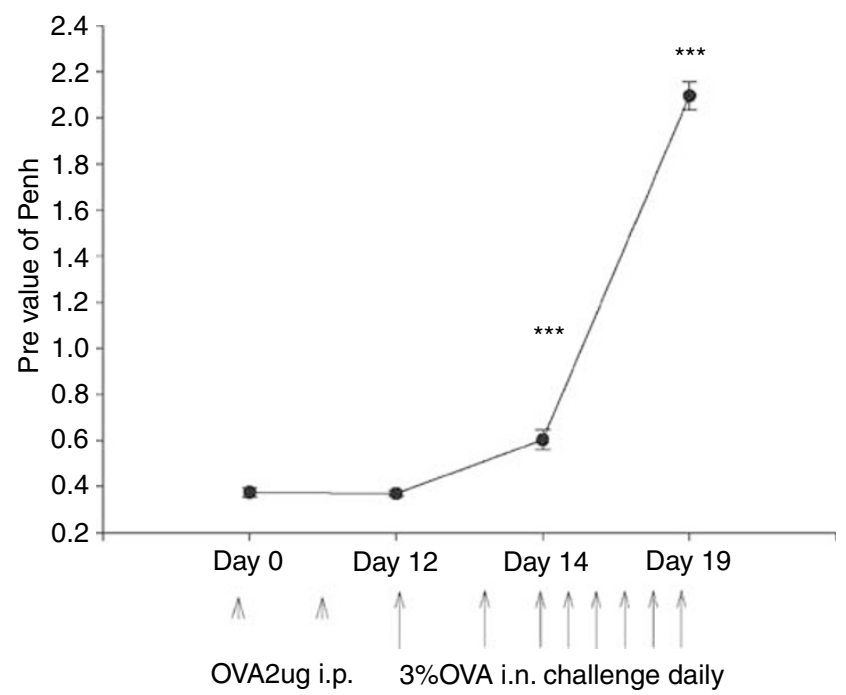

Figure 2 Serial change in Penh following sensitization and nasal challenge.

that airflow limitation in the nasal passage could affect the Penh value without affecting the lower respiratory tract. Next, we examined serial changes in the Penh value following the development of nasal allergic inflammation.

\section{Change in Penh Following Sensitization and Nasal Challenge}

Penh was measured just before systemic OVA sensitization or OVA nasal challenge at each experimental period. Along with the development of nasal allergic inflammation, Penh gradually increased (Figure 2). The mean values of Penh on days 14 and 19 were significantly higher compared with those on day $0(P<0.001$, respectively). 


\section{Change in Serum IgE and OVA-Specific IgE}

Figure 3a shows the change in the serum IgE concentration at each experimental time point $(n=8)$. In the control group, no significant change in $\operatorname{IgE}$ was detected during the experiment. In the OVA-treated group, IgE increased gradually following the time period. There were significant differences between the IgE value on day 0 , and those values on days 12,14 , and 19 (day $12, P<0.05$; days 14 and $19, P<0.01)$. Figure $3 b$ shows a change in the serum OVA-specific concentration at each experimental time point $(n=8)$. In the control group, serum OVA-specific IgE was not detected during the period of the experiment. In the OVA-treated group, OVA-specific IgE increased gradually following sensitization. There were significant differences between the OVA-specific value on day 0 and those on days 12,14 , and 19, respectively, the same as the change in serum IgE (day $12, P<0.05$; days 14 and 19, $P<0.01)$.

\section{Eosinophil Infiltration in Nasal Mucosa}

Figure 4a shows the pathological findings of nasal septal mucosa. Nasal mucosa on day 0 showed no
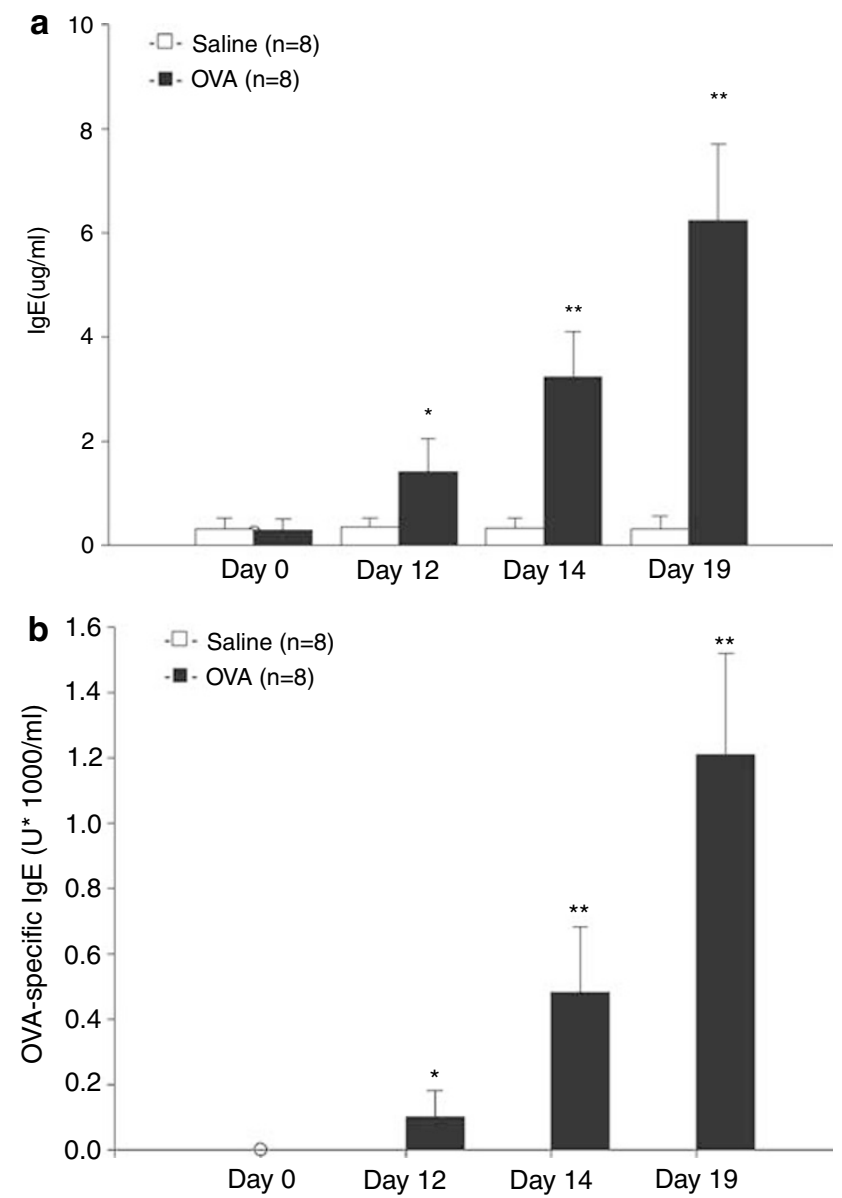

Figure 3 The changes in serum IgE (a) and OVA-specific IgE (b) concentration at each experimental point. infiltration of inflammatory cells, but on day 12 showed a slight infiltration. Furthermore, nasal mucosa on day 14 showed a moderate infiltration of inflammatory cells, and on day 19 showed an accumulation of inflammatory cells. The mean number of eosinophils in the submucosa gradually increased following sensitization and nasal challenge (Figure 4b). The mean number of eosinophils in submucosa on day 12 was $2.0 \pm 0.62$ (mean value \pm s.e.m.), and there was no significant difference compared with that on day 0 . The mean number of eosinophils on day 14 was $5.25 \pm 0.88$, and there was a significant difference compared with that on day $0(P<0.05)$. There was a rapid increase in the eosinophil number from day 14 to day $19(18.88 \pm 1.46)$. A statistically significant difference was detected from day 0 to day 14 $(P<0.05)$ and between day 0 and day $19(P<0.01)$.

\section{Allergen-Induced Nasal Symptoms}

Nasal allergic symptoms induced by allergen delivery, such as sneezing and nasal itching, accelerated significantly as a result of daily intranasal challenge. Figure 5a shows the mean number of sneezes/10 min after the intranasal challenge at each experience time $(n=8)$. The mean number of sneezes on day 12 was not significantly different compared with the number on day 0 . The mean numbers of sneezes on days 14 and 19 were significantly greater than on day $0(P<0.001)$. Figure 5 b shows the mean number of scratches/10 min after intranasal challenge. The mean number of scratches on day 12 was not significantly different compared with day 0 . The mean number of sneezes on day 14 or day 19 was significantly greater than on day $0(P<0.001)$. Taken together, these results indicate that an increase in Penh value strongly correlated with changes in various parameters characteristic for experimental allergic rhinitis (Figures 2-5).

\section{Serial Change in Penh in Immediate-Type Hypersensitivity Reaction in the Nasal Airway}

We further extended our study to elucidate whether the Penh system could be applicable to an analysis of immediate-type hypersensitivity in the nasal airway. OVA nasal challenge was carried out on day 19; then serial change in Penh was measured for about $40 \mathrm{~min}$ (Figure 6). Immediately after the OVA delivery (1 min), Penh rapidly decreased, probably because of an almost complete obstruction of nasal cavities with the OVA solution. Penh soon recovered to the baseline level in $1 \mathrm{~min}$, and then rapidly increased within a few minutes. Its maximal mean value was $2.93 \pm 0.27$, significantly greater than the prechallenge value $(P<0.005)$. After the rapid increase, Penh gradually increased for approximately $20 \mathrm{~min}$. Its maximal mean value of Penh was $3.70 \pm 0.27$, observed at $25 \mathrm{~min}$ after nasal 

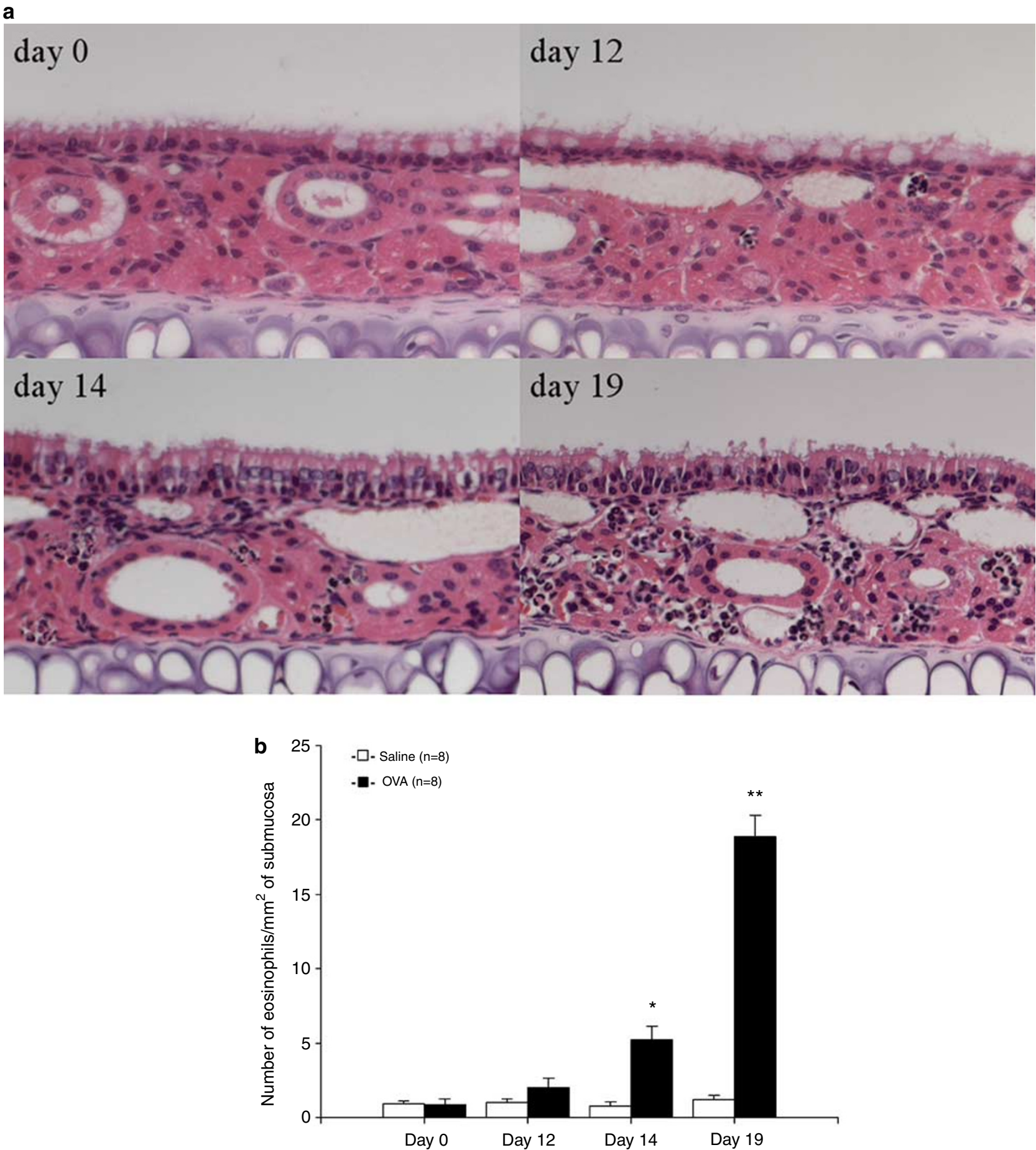

Figure 4 Histological change and infiltration of eosinophils into nasal mucosa. (a) Histological findings of nasal septal mucosa $(\mathrm{H}-\mathrm{E}, \times 100)$. (b) Infiltration of eosinophils into nasal mucosa. ${ }^{*} P<0.05$ and ${ }^{*}{ }^{*} P<0.01$.

challenge (vs base line value; $P<0.0001$ ). Penh then slightly decreased. Pretreatment of the sensitized mice with dexamethasone or a histamine $\mathrm{H} 1$ blocker induced a slight decrease in baseline Penh value at the time of OVA provocation. In dexamethasonetreated mice, Penh rapidly increased within a few minutes after the OVA provocation, but began to decrease shortly after it reached a maximal value (Figure 6). The Penh continued to decline in this group thereafter. In contrast, in the group of mice that received the $\mathrm{H} 1$ blocker, the rapid increase in Penh was not observed. Moreover, Penh remained at a low level throughout the experiment.

\section{Bronchoalveolar Lavage Analysis}

To elucidate whether inflammation of the lung would be induced in this murine allergic model, 

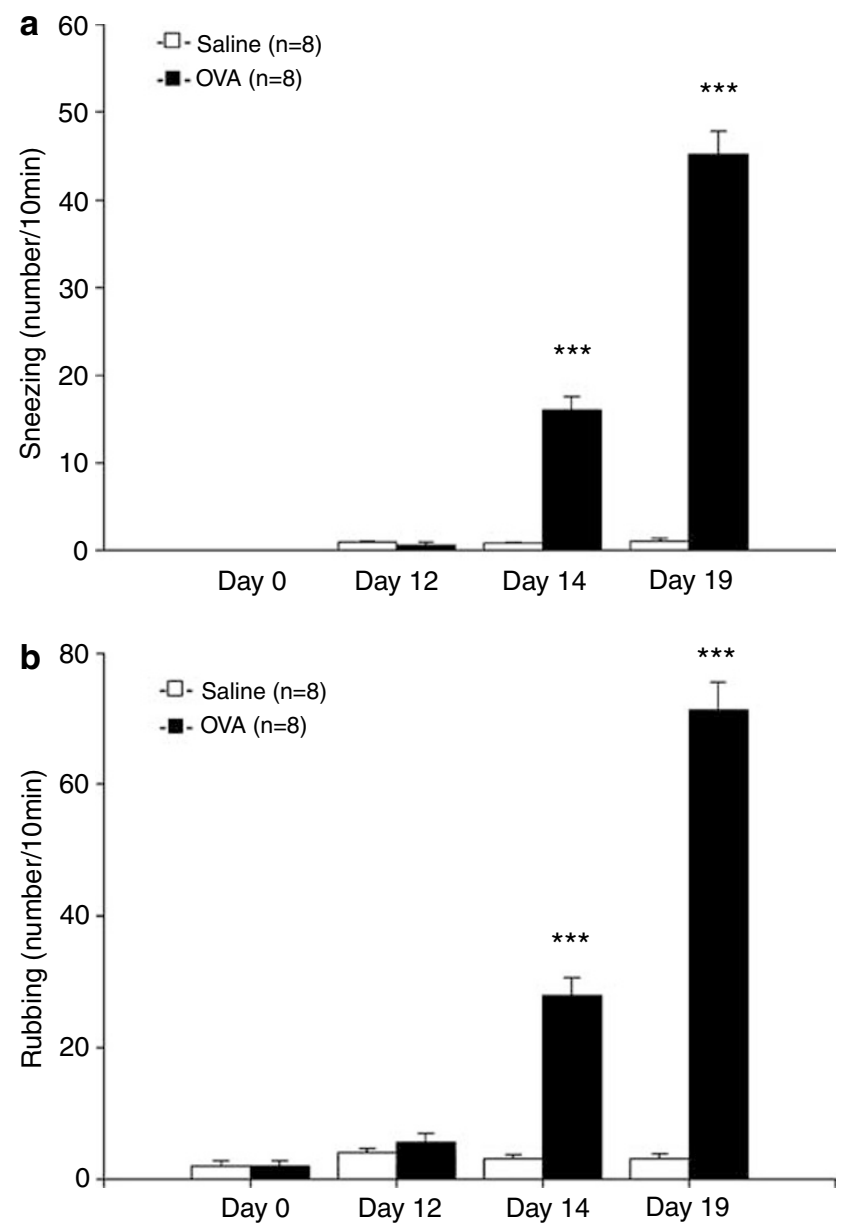

Figure 5 The mean number of sneezing (a) and rubbing (b) after nasal challenge.

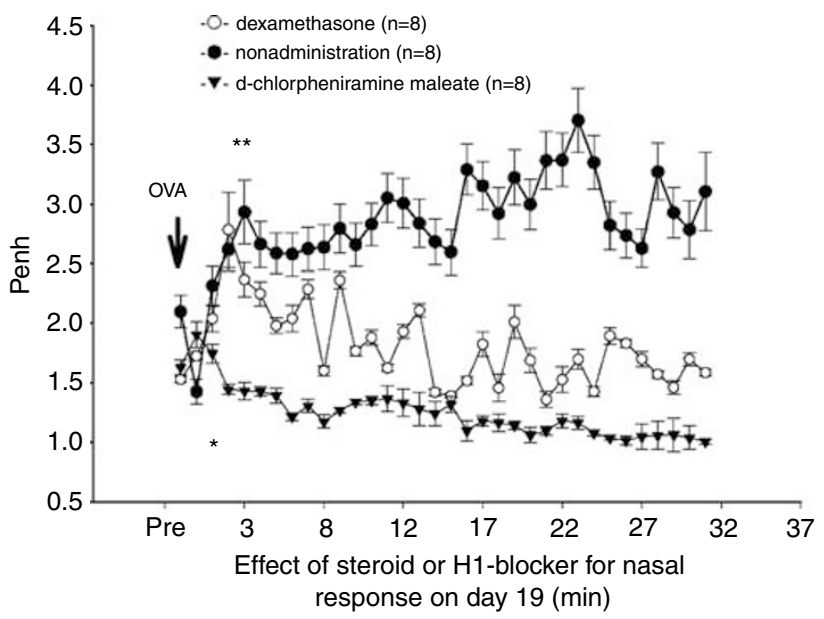

Figure 6 Serial changes in Penh after nasal challenge.

we analyzed bronchoalveolar lavage fluid (BALF) findings following the sensitization (Figure 7). In the control group and OVA-treated group, no significant changes in the cell number or cell differentials were observed throughout the time period and there was no significant difference among both groups.

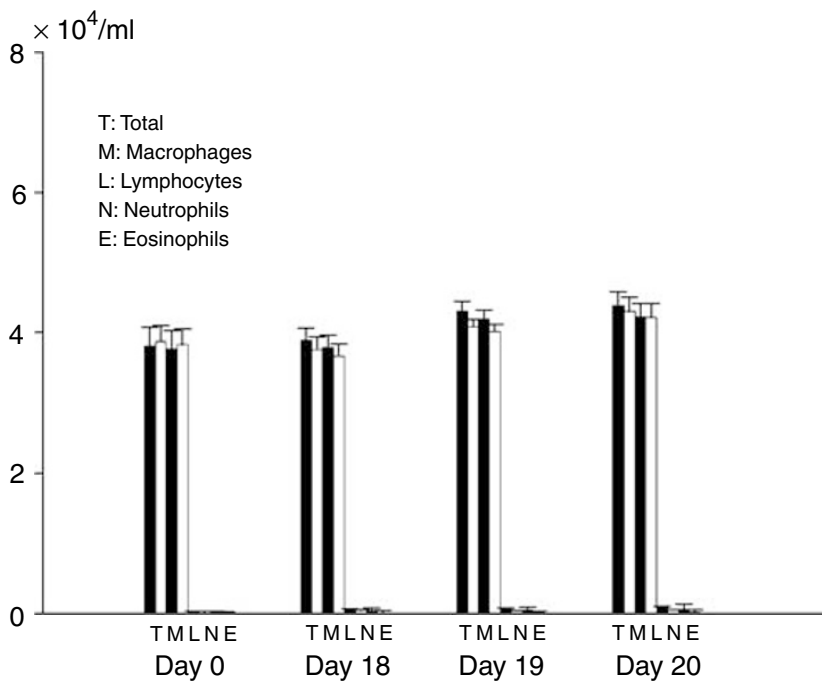

Figure 7 Change in BALF differential cell count following sensitization.

\section{Airway Resistance of the Lower Respiratory Tract and} Lung Tissue after Nasal OVA Challenge

The average Raw before nasal allergen challenge was $1.45\left(\mathrm{cmH}_{2} \mathrm{O} / \mathrm{ml} / \mathrm{s}\right) ; 20 \mathrm{~min}$ after nasal challenge it was $1.46\left(\mathrm{cmH}_{2} \mathrm{O} / \mathrm{ml} / \mathrm{s}\right)$; and $24 \mathrm{~h}$ after nasal challenge it was $1.39\left(\mathrm{cmH}_{2} \mathrm{O} / \mathrm{ml} / \mathrm{s}\right)$ (Figure 8a). There was no significant difference between timepoints. Figure $8 \mathrm{~b}$ shows the histopathology of lung tissue before and after the OVA nasal challenge. There was a slight degree of infiltration of inflammatory cells into the bronchial tissue in the mouse before the challenge (left), $20 \mathrm{~min}$ after the challenge (center), and $24 \mathrm{~h}$ after the challenge (right). The intensity of cell infiltration was not different among the three groups. Furthermore, apparent bronchoconstriction was not observed in either group. These results confirmed that the increase in Penh after the nasal allergen challenge was not induced by allergeninduced broncoconstriction in the lower respiratory tract, but by the immediate-type hypersensitivity reaction in the nasal airway in our experimental model of allergic rhinitis.

\section{Discussion}

The results of this study clearly demonstrate that the measurement of Penh would be a very useful tool for analyzing the immediate-type hypersensitivity reaction in the nasal airway. Change in Penh during the development of nasal allergy strongly correlated with various indicators of nasal allergic reaction, such as IgE production, eosinophil recruitment in nasal tissue, and allergen-induced symptoms. Moreover, Penh precisely reflected the acute response induced by allergen provocation, and also reflected the effect of drug treatment. So far, the physiological measurement of nasal allergy has been restricted to 

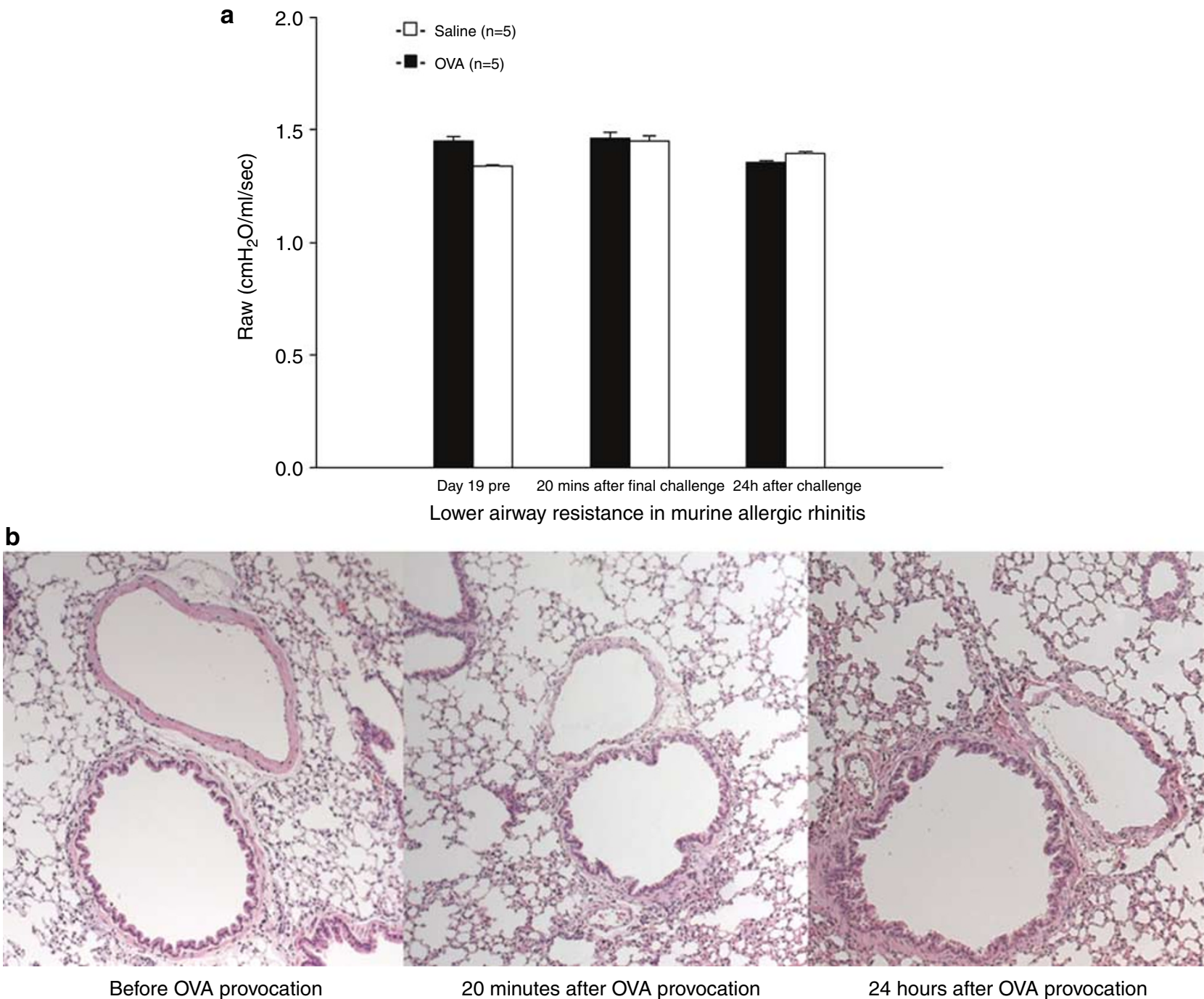

Figure 8 Effect of nasal challenge on the lower respiratory tract. (a) Respiratory resistance of the lower respiratory tract. (b) Histological findings of the lung before and after OVA challenge $(\mathrm{H}-\mathrm{E}, \times 100)$.

invasive procedures ${ }^{7,8}$ or to the use of larger animals such as cats ${ }^{9}$ or dogs. ${ }^{10}$ There has been no reliable method for measuring physiological change in the nasal airway in mice. This is the first report demonstrating physiological changes in nasal airway in a conscious, noninvasive condition in mice.

Hamelmann et $a l^{11}$ reported that Penh correlates well with the pulmonary resistance measured with conventional two-chamber plethysmography in anesthetized, unconscious animals. Penh has been widely used for measuring nonspecific bronchial hyper-responsiveness in murine models of allergic airway inflammation. ${ }^{11-15}$ However, recent studies have suggested that there are problems with the Penh system; that it does not necessarily correlate with airway resistance. Albertine et $a l^{16}$ and Petak et $a 1^{17}$ have shown that there is an inconsistent relationship between Penh and invasive measurements, especially in C57BL/6 mice. Mitzner et $a l,{ }^{23,24}$ Lundblad et $a l^{25}$ and Enhorning et $a l^{26}$ have shown that the relationship between the chamber pressure, from which Penh is calculated, and the airway resistance is limited. A reason for this dissociation may be attributed to the method to measure Penh. Because Penh is a dimensionless marker that just represents a change in the pressure of the box chamber caused by airflow limitation, only the changes in the lower airway could not always determine its value. Airflow limitation in the upper airway, including the nasal cavity, might affect the value. Our results clearly confirmed this possibility. The present findings suggest that this noninvasive indicator still could be used widely, but to evaluate the obtained result correctly, we must be careful in regard to the experimental system.

First we examined whether nasal congestion induced by histamine could affect the Penh value. After an intranasal challenge of histamine, Penh increased without lower airway constriction in naive mice, and it decreased with a relief of congestion with the administration of epinephrine (Figure 1). Tiniakov et $a{ }^{10}$ described a canine model 
of nasal congestion and allergic rhinitis by anterior rhinomanometry and acoustic rhinometry. They showed that histamine-induced increases in resistance to airflow and phenylephrine ( $\alpha$-adrenomimetics) caused a rapid relief of the nasal congestion in nonsensitized canine. In their study, the maximal increase in resistance was about eight times greater than the baseline level. In our study, the maximal increase in Penh was about 2.5 times compared with that of the baseline value before nasal challenge (Figure 1). The difference in the data may be due to the difference between animals. In canine nasal mucosa, blood vessels are more abundant than those in mice nasal mucosa. ${ }^{27}$ However, a rapid relief of the nasal congestion caused by $\alpha$-adrenomimetics in nonsensitized canine was similar to our result with mice (Figure 1). These data indicate that Penh could change by congestion or contraction in nasal mucosa of mice without pathological change in the lower airway and thus would reflect nasal hypersensitivity in mice.

Next, we investigated allergen-specific nasal hypersensitivity by the Penh system in a murine model of allergic rhinitis. After systemic sensitization, the intranasal allergen challenge induced an infiltration of eosinophils into nasal mucosa (Figure 4). Serum IgE and OVA-specific IgE were also produced (Figure 3). Penh did not increase by systemic sensitization alone. With intranasal challenge, Penh gradually increased from day 12 to day 14 , and increased further along with repeated allergen challenges from day 14 to day 19 (Figure 2 ). The increase in eosinophils in nasal musosa and serum IgE correlated very well with the increase in Penh value. Furthermore, the intensity of allergic symptoms also correlated well with Penh (Figure 5). Therefore, Penh could be a good indicator to monitor the development of allergic inflammation in murine nasal mucosa.

Finally, we elucidated whether the Penh system could be applicable to an analysis of immediate-type hypersensitivity in the nasal airway. Serial changes in Penh value were followed after intranasal OVA provocation in sensitized mice (Figure 6). We confirmed that an intranasal delivery of OVA solution did not induce bronchoconstriction in the lower airway tract; nor did OVA challenge induce an increase in airway resistance in the sensitized mouse (Figure 8a). These findings were supported by BALF (Figure 7) and pathological findings (Figure 8b). In a previous study using murine allergic rhinitis, the quantity of allergen solution administered intranasally was $10 \sim 60 \mu \mathrm{l}$ per times. ${ }^{28,29}$ We confirmed that nasal drip of $40 \mu \mathrm{l}$ blue liquid cannot reach the trachea in the unanesthetized mice (data not shown). Therefore, unanesthetized mice would swallow the rest of allergen without aspiration after nasal drip; only anesthetized mice would inhale all of the liquid allergen. It should be noted that in murine asthma models, aerosol of allergen was used widely, because inhaled particles reach the lung. Therefore, changes in Penh were considered to reflect changes in the nasal airway induced by allergen-specific nasal responses.

In this study, Penh showed a transient decrease just after the intranasal delivery of OVA solution, probably because of a transient, but severe, obstruction of the nasal cavity (Figure 6). Subsequently, Penh increased rapidly within 5 min. After this acute phase, it continued to increase slightly and reached a maximal value at $25 \mathrm{~min}$ after the provocation. Treatment of the sensitized mice with dexamethasone could not suppress the rapid increase in Penh, whereas a histamine $\mathrm{H} 1$ blocker (d-chlorpheniramine maleate) completely suppressed it. This finding strongly suggests that the main mediator involved in this acute phase is histamine. Although the acute increase was not suppressed, with dexamethasone treatment Penh began to decline after it reached a peak and continued to decline thereafter. The gradual increase observed in the nontreated group of mice between the points of 5 and $25 \mathrm{~min}$ was suppressed. In contrast, the effect of histamine H1 blocker continued throughout the experiment. The therapeutic effect of d-chlorpheniramine maleate is mediated not only by histamine $\mathrm{H} 1$ receptor antagonism but also by inhibition of the release of both preformed mediators, such as histamine, and de novo synthesized mediators, such as leukotrienes and prostaglandins from mast cells and basophils. ${ }^{30}$ Furthermore, glucocorticosteroids suppress the generation of IL-3, IL-5, and GM-CSF by T lymphocytes, leading to a deprivation of the eosinophil survival cytokines, ${ }^{31,32}$ and they directly inhibit the prolongation of eosinophil survival by these cytokines. ${ }^{33,34}$ These data suggest that in the second phase, when Penh gradually increased, numerous mediators other than histamine, such as leukotrienes, prostaglandins, and interleukins, would be involved in the inflammation in the nasal airway, which should be further investigated.

Mice offer many advantages as an experimental animal for the study of nasal allergy. With mice, it is easy to conduct such manipulations as antibody administration, cell transfer, gene transfer, and genetic interventions. Many studies accomplished with these techniques in mice have already been reported, providing much information for the study of nasal allergy. Our results suggest that a combination of these manipulations and the noninvasive Penh system should facilitate the elucidation of the pathophysiology of nasal allergy. Further, Penh would be extremely useful and convenient for an evaluation of the effect of therapeutic agents. Further, this system would also be useful for studying nasal responses other than the immediate-type reaction, such as late phase reaction, and should be investigated in the future. 


\section{References}

1 Wihl JA, Malm L. Rhinomatometry and nasal peak expiratory and inspiratory flow rate. Ann Allergy 1988;61:50-55.

2 Widdicombe JG. Nasal pathophysiology. Resp Med 1990;54:3-10.

3 Panagou P, Loukides S, Tsipra S, et al. Evaluation of nasal patency: comparison of patient and clinician assessments with rhinomanometry. Acta Otolaryngol 1998;118:847-851.

4 Oluwole M, Gardiner Q, White PS. The naso-oral index: a more valid measure than peak flow rate? Clin Otolaryngol 1997;22:346-349.

5 Nathan RA, Eccles R, Howarth PH, et al. Objective monitoring of nasal patency and nasal physiology in rhinitis. J Allergy Clin Immunol 2005;115:S442-S459.

6 Hilberg O. Objective measurement of nasal airway dimensions using acoustic rhinometry: methodological and clinical aspects. Allergy 2002;57:5-39.

7 Albert $\mathrm{DH}$, Malo PE, Tapang $\mathrm{P}$, et al. The role of palatelet-activatin factor (PAF) and the efficacy ABT491, a highly potent and selective PAF antagonist, in experimental allergic rhinitis. J Pharmacol Exp Ther 1998;284:83-88.

8 McLeod RL, Young SS, Erickson CH, et al. Characterizatin of nasal obstruction in the allergic guinea pig using the forced oscillation method. J Pharmacol Toxixol Methods 2002;48:153-159.

9 McLeod RL, Mingo GG, Herczku C, et al. Changes in nasal resistance and nasal geometry in a feline model of nasal congestion. Am J Rhinol 1999;13:375-383.

10 Tiniakov RL, Tiniakova OP, McLeod RL, et al. Canine model of nasal congestion and allergic rhinitis. J Appl Physiol 2003;94:1821-1828.

11 Hamelmann E, Schwartz J, Takeda K, et al. Noninvasive measurement of airway responsiveness in allergic mice using barometric plethysmography. Am J Resp Crit Care Med 1997;156:766-775.

12 Lee JJ, McGarry MP, Farmer SC, et al. Interleukin-5 expression in the lung epithelium of transgenic mice leads to pulmonary changes pathognomonic of ashma. J Exp Med 1997;185:2143-2156.

13 Schwarze J, Hamelmann E, Bradley KL, et al. Respiratory syncytial virus infection results in airway hyper responsiveness and enhanced airway sensitization to allergen. J Clin Invest 1997;100:226-233.

14 Kline JN, Waldschmidt TJ, Businga TR, et al. Modulation of airway inflammation by CpG oligonucleotides in a murine model of asthma. J Immunol 1998;160: $2555-2559$

15 Nakagome K, Dohi M, Okunishi K, et al. In vivo IL-10 gene delivery suppresses airway eosinophilia and hyperreactivity by down-regulating APC functions and migration without impairing the antigen-specific systemic immune response in a mouse model of allergic airway inflammation. J Immunol 2005;174: 6955-6966.

16 Albertine $\mathrm{KH}$, Wang L, Watanabe S, et al. Temporal correlation of measurements of airway hyperresponsiveness in ovalbumin-sensitized mice. Am J Physiol Lung Cell Mol Physiol 2002;283:L219-L233.

17 Petak F, Habre W, Donati YR, et al. Hyperoxia-induced changes in mouse lung mechanics: forced oscillations vs barometric plenthysmography. J Appl Physiol 2001;90:2221-2230.

18 Jacky JP. A plethysmograph for long-term measurement of ventilation in unrestrained animals. J Appl Physiol 1978;45:644-647.

19 Nakagome K, Dohi M, Okunishi K, et al. Antigensensitized CD4+CD62L low memory/effector T helper 2 cells can induce airway hyperresponsiveness in an antigen free setting. Resp Res 2005;28:46.

20 To Y, Dohi M, Tanaka R, et al. Early interleukin 4dependent response can induce airway hyperreactivity before development of airway inflammation in amouse model of athma. Lab Invest 2001;81:1385-1396.

21 Okunishi K, Dohi M, Nakagome K, et al. A novel role of cysteinyl leukotrienes to promote dendritic cell activation in the antigen-induced immune resposes in the lung. J Immunol 2004;173:6393-6402.

22 Hussain I, Jain VV, Kitagaki K, et al. Modulation of murine allergic rhinosinusitis by $\mathrm{CpG}$ oligodeoxynucleotides. Laryngoscope 2002;112:1819-1826.

23 Mitzner W, Tankersley C. Noninvasive measurement of airway responsiveness in allergic mice using barometric plethysmography. Am J Resp Crit Care Med 1998;158:340-341.

24 Mitzner W, Tankersley C, Lundblad LKA, et al. Interpreting Penh in mice. J Appl Physiol 2003;94: 828-832.

25 Lundblad KKA, Irvin CG, Adler A, et al. A reevaluation of the validity of unrestrained plethysmography in mice. J Appl Physiol 2002;93:1198-1207.

26 Enhornign G, Van Schaik S, Lundgren C, et al. Wholebody plethysomograhpy, does it measure tidal volume of small animals? Can J Physiol Pharmacol 1998; 76:945-951.

27 Lung MA, Phipps RJ, Wang JCC, et al. Control of nasal vasculature and airflow in the dog. J Physiol 1984;349:535-551.

28 Yamamoto K, Kawamura I, Tominaga T, et al. Listeriolysin $\mathrm{O}$ derived from Listeria monocytogenes inhibits the effector phase of an experimental allergic rhinitis induced by ovalbumin in mice. Clin Exp Immunol 2006;144:475-484.

29 Farraj AK, Harkema JR, Kaminski NE. Allergic rhinitis induced by intranasal sensitization and challenge with trimellitic anhydride but not with dinitrochlorobenzene or oxazolone in A/J mice. Toxicol Sci 2004; 79:315-325.

30 Assanasen P, Naclerio RM. Antiallergic anti-inflammatory effects of H1-antihistamines in humans. Clin Allergy Immunol 2002;17:101-139.

31 Guyre PM, Girard MT, Morganelli PM, et al. Glucocorticoid effects on the production and actions of immune cytokines. J Steroid Biochem 1988;30:89-93.

32 Leung DY, Martin RJ, Szefler SJ, et al. Dysregulation of interleukin 4 , interleukin 5 , and interferon gamma gene expression in steroid-resistant asthma. J Exp Med 1995;181:33-40.

33 Lamas AM, Leon OG, Schleimer RP. Glucocorticoids inhibit eosinophil responses to granulocyte-macrophage colony-stimulating factor. J Immunol 1991;147: 254-259.

34 Wallen N, Kita H, Weiler D, et al. Glucocorticoids inhibit cytokine-mediated eosinophil survival. J Immunol 1991;147:3940-3945. 